\title{
EFEITOS DA TERMORRETIFICAÇÃO NA PERDA DE MASSA E PROPRIEDADES MECÂNICAS DE Eucalyptus grandis E Pinus caribaea VAR. hondurensis
}

\author{
Luiz Fernando de Moura ${ }^{1}$, José Otávio Brito ${ }^{1}$, Geraldo Bortoletto Júnior ${ }^{1}$ \\ ${ }^{1}$ Eng. Florestal, Dr., Depto. de Ciências Florestais, ESALQ/USP, Piracicaba, SP, Brasil - demoura.lf@usp.br; jobrito@usp.br; \\ gbortoll@esalq.usp.br \\ Recebido para publicação: 04/06/2010 - Aceito para publicação: 23/12/2011
}

\begin{abstract}
Resumo
A termorretificação é um processo de agregação de valor, que confere à madeira colorações semelhantes àquelas de espécies tropicais de maior valor econômico, além de melhorar sua estabilidade dimensional e resistência a fungos. A termorretificação deve ser estudada visando obter seus benefícios com o mínimo de perdas referentes às propriedades mecânicas da madeira. Madeiras consideradas de menor valor econômico (Eucalyptus grandis e Pinus caribaea var. hondurensis) foram submetidas a diversos tratamentos de termorretificação, em presença de oxigênio do ar (140, 160 e $\left.180^{\circ} \mathrm{C}\right)$ e escassez de oxigênio $(160,180$ e $200{ }^{\circ} \mathrm{C}$ ), sendo analisadas suas propriedades mecânicas. O eucalipto, além de ter sido mais susceptível à perda de massa com o aumento da temperatura, também manifestou maior propensão à perda de resistência em função do tratamento térmico, quando comparado ao pínus. A termorretificação resultou em queda da resistência ao cisalhamento, para ambas as madeiras testadas, e à flexão estática apenas para a madeira de eucalipto. A temperatura de $200{ }^{\circ} \mathrm{C}$ não foi suficiente para provocar perda da resistência à compressão paralela. Não foi possível determinar com clareza o efeito das condições de presença e escassez de oxigênio do ar durante a termorretificação sobre as propriedades mecânicas da madeira.

Palavras-chave: Tratamento térmico; resistência mecânica; madeira.
\end{abstract}

\begin{abstract}
Effects of thermal rectification on mass loss and mechanical properties of Eucalyptus grandis and Pinus caribaea var. hondurensis woods. The thermal rectification is an adding-value process, which provides to wood colors similar to those observed in high-valued tropical species, as well as higher dimensional stability and better resistance against fungi. The thermal rectification process must be studied seeking to obtain its benefits with minimum losses in mechanical properties of wood. Comparatively low-valued woods (Eucalyptus grandis and Pinus caribaea var. hondurensis) had been submitted to different thermal rectification treatments, in presence of oxygen from the air $\left(140,160\right.$ and $\left.180{ }^{\circ} \mathrm{C}\right)$ and scarcity of oxygen $\left(160,180\right.$ and $200{ }^{\circ} \mathrm{C}$ ), and their mechanical properties were evaluated. Eucalyptus was more susceptible to mass loss with increasing temperature, and was more prone to loss of mechanical resistance during thermal treatments, compared to pinus. The thermal rectification reduced shear strength, for both species tested, as well as bending strength, for eucalyptus only. The treatment at 200 ${ }^{\circ} \mathrm{C}$ was not sufficient to cause losses in longitudinal compressive strength. It was not possible to clearly determine effects of presence or scarcity of air oxygen during thermal rectification on the mechanical properties of wood.
\end{abstract}

Keywords: Thermal treatment; mechanical resistance; wood.

\section{INTRODUÇÃO}

A termorretificação é um tipo de tratamento térmico que visa aumentar a durabilidade natural e melhorar a aparência de madeiras de menor valor econômico, alterando as características físicas da madeira. A madeira termorretificada adquire colorações semelhantes àquelas de madeiras tropicais de maior valor econômico agregado, considerável resistência a fungos xilófagos e à ação climática, alta estabilidade dimensional e baixa higroscopicidade (DUCHEZ; GUYONNET, 1998). No entanto, esse tratamento térmico pode impactar negativamente algumas propriedades mecânicas da madeira (STAMM, 
1956; KAMDEM et al., 2002; BEKHTA; NIEMZ, 2003).

Alguns estudos indicam um efeito significativo da temperatura sobre o módulo de ruptura das madeiras tratadas termicamente, comparado a uma menor influência da temperatura sobre o módulo de elasticidade. Para a madeira de Picea abies aquecida a temperaturas até $200{ }^{\circ} \mathrm{C}$, Bekhta e Niemz (2003) observaram uma redução média do módulo de ruptura da ordem de 44 a 50\%, enquanto o módulo de elasticidade foi reduzido em apenas 4 a $9 \%$.

Stamm (1956), para um tratamento térmico durante 30 minutos a $200^{\circ} \mathrm{C}$, relatou que amostras de coníferas apresentaram redução de $10 \%$ do módulo de ruptura e $1 \%$ da massa. Kamdem et al. (2002) estudaram o processo francês "Bois Rectifié" e constataram perdas de 10 a 50\% no Módulo de Ruptura (MOR) em flexão estática. Tsoumis (1991) apresentou um estudo com a madeira de Pinus sylvestris, no qual o aumento da temperatura do tratamento térmico causou redução do módulo de elasticidade. No tratamento térmico de Betula sp. durante 2 e 4 horas, a 180 e $200{ }^{\circ} \mathrm{C}$, Awoyemi e Westermark (2005) constataram redução no módulo de elasticidade e módulo de ruptura em flexão estática.

Kubojima et al. (2000) estudaram os efeitos do tratamento térmico em Picea sitchensis a temperatura máxima de $160^{\circ} \mathrm{C}$ em presença e escassez de oxigênio, durante períodos variando de 0,5 a 16 horas. Os autores verificaram que os módulos de elasticidade e ruptura em flexão estática aumentaram no estágio inicial do tratamento térmico e decresceram em seguida, tendo diminuído mais na presença do que na escassez de oxigênio. $O$ trabalho necessário para ruptura diminuiu em função do aumento do tempo de aquecimento, tendo decrescido mais na ausência que na presença de oxigênio. Esses resultados indicam a dificuldade de interpretação do real efeito da presença ou ausência de oxigênio durante o tratamento térmico. Os autores relatam que a madeira tratada termicamente tornou-se mais quebradiça nos testes de flexão estática, em comparação às testemunhas não tratadas.

Santos (2000) constatou um aumento do módulo de elasticidade em flexão estática póstratamento térmico na madeira de Eucalyptus globulus tratada pelo processo francês de termorretificação "Bois Rectifiee", comparada às testemunhas não tratadas.

Diversas propriedades físicas e mecânicas de Pinus taeda foram estudadas após o tratamento a $150{ }^{\circ} \mathrm{C}$ por 16 horas, em duas atmosferas diferentes, uma com injeção de nitrogênio (inerte), e outra com oxigênio do ar. Para a temperatura testada, o aquecimento da madeira em atmosfera com oxigênio do ar praticamente não teve efeito degradativo adicional sobre o módulo de ruptura, comparado ao aquecimento em nitrogênio (MITCHELL, 1988). Isso sugere que, dependendo da dimensão das amostras tratadas termicamente e da temperatura máxima adotada, a proporção de oxigênio na atmosfera de tratamento torna-se um fator de importância secundária.

Existem evidências de que a eliminação dos carboidratos durante o tratamento térmico seja a principal causa de redução da resistência mecânica em madeiras tratadas. Um modelo matemático linear elaborado por Winandy e Lebow (2001), utilizando hemiceluloses (compostas por arabinose e manose) e glicose como indicador da intensidade de degradação da celulose, foi capaz de predizer a perda de resistência mecânica da madeira tratada termicamente $\left(R^{2}>0,75\right)$.

As referências literárias indicam a necessidade de estudar o processo de termorretificação visando obter os benefícios desse tratamento térmico com o mínimo de perdas em relação às propriedades mecânicas da madeira tratada. Nesse contexto, o presente trabalho teve como objetivo elucidar o efeito da termorretificação nas resistências à flexão estática, compressão paralela e cisalhamento das madeiras de Eucalyptus grandis e Pinus caribaea var. hondurensis tratadas em diversas temperaturas máximas (140, 160,180 e $200^{\circ} \mathrm{C}$ ), em presença e escassez de oxigênio na atmosfera do interior da câmara de tratamento térmico. As espécies escolhidas para este estudo são muito usadas no Brasil em plantios florestais e são consideradas de importante potencial para suprir a demanda da indústria madeireira nacional.

\section{MATERIAL E MÉTODOS}

\section{Procedência e obtenção das madeiras}

Foram estudadas as madeiras de Eucalyptus grandis (18 anos, proveniente de Anhembi, SP) e Pinus caribaea var. hondurensis (32 anos, proveniente de Uberlândia, MG). As densidades básicas médias das pranchas não tratadas eram de 532 e $394 \mathrm{~kg} \cdot \mathrm{m}^{-3}$, para E. grandis e $P$. caribaea var. hondurensis, respectivamente. Pranchas de $280 \times 8 \times 5 \mathrm{~cm}$ foram cortadas em quatro subamostras de $60 \times 8 \times 5 \mathrm{~cm}$, eliminando-se zonas defeituosas (rachaduras, nós e empenamentos). Para cada prancha, uma das subamostras foi usada em seu estado natural, como testemunha, e as outras três destinadas aos 
tratamentos térmicos. As amostras foram divididas em dois grupos, em função da atmosfera testada durante as termorretificações: para amostras tratadas em presença de oxigênio do ar, as subamostras foram destinadas às temperaturas máximas de $140{ }^{\circ} \mathrm{C}, 160{ }^{\circ} \mathrm{C}$ e $180{ }^{\circ} \mathrm{C}$ (além da testemunha); para amostras tratadas em escassez de oxigênio, as subamostras foram destinadas às temperaturas máximas de $160{ }^{\circ} \mathrm{C}, 180{ }^{\circ} \mathrm{C}$ e $200{ }^{\circ} \mathrm{C}$ (além da testemunha). As peças selecionadas para as retificações térmicas (sete amostras por tratamento) foram secas em estufa com circulação forçada de ar à temperatura de $100{ }^{\circ} \mathrm{C}$, até atingirem massa constante, conforme indicado por Pincelli et al. (2002).

\section{Procedimento de termorretificação das madeiras}

As retificações térmicas foram conduzidas nos Laboratórios Integrados de Química, Celulose e Energia (LQCE) do Departamento de Ciências Florestais da Escola Superior de Agricultura "Luiz de Queiroz", Universidade de São Paulo (ESALQ/USP). As madeiras foram termorretificadas em estufa com sistema de aquecimento por resistência elétrica e circulação forçada de ar. As madeiras foram colocadas inicialmente no interior de caixas metálicas fechadas (Figura 1), com porta de acesso e orifícios destinados à colocação da fiação de sistema de controle de temperatura, acesso para evacuação de gases do processo e a introdução de gases para inertização da atmosfera interna, quando prevista (Figuras 1A e 1B). As caixas contendo as madeiras foram introduzidas na estufa com a temperatura pré-regulada a $100{ }^{\circ} \mathrm{C}$ e, em seguida, foi iniciada a termorretificação, conforme os programas apresentados na figura 2.
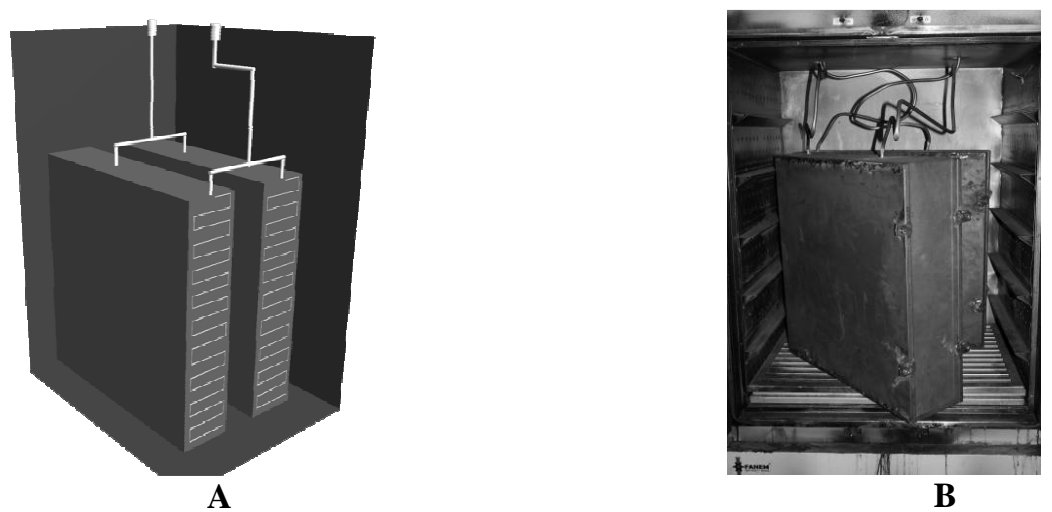

Figura 1. Projeto das caixas para instalação no interior da estufa, visando injeção de nitrogênio gasoso: A) Tubulação à direita para entrada do gás, tubulação à esquerda para a saída do gás; B) Visão geral do sistema instalado na estufa.

Figure 1. Thermal rectification chambers designed to nitrogen injection, installed into the oven: A) Right-hand pipeline for gas entry, left-hand pipeline for gas purge; B) Overview of the system installed inside the oven.

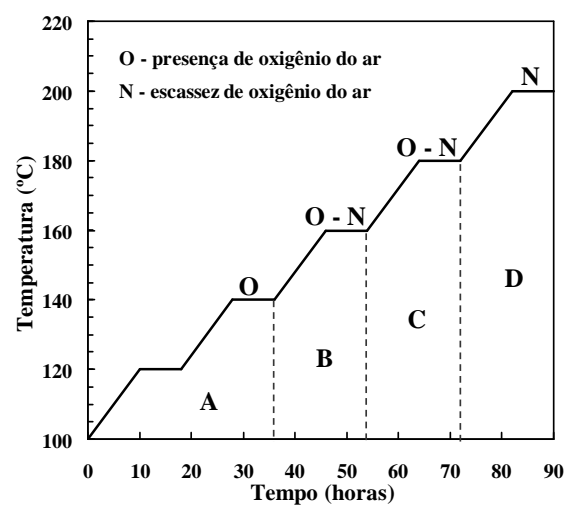

Figura 2. Programas usados para termorretificações a temperaturas máximas de $140,160,180$ e $200{ }^{\circ} \mathrm{C}$. Figure 2. Thermal rectification programs reaching maximum temperatures of $140,160,180$ and $200^{\circ} \mathrm{C}$. 
Para testar o efeito da escassez de oxigênio na câmara de termorretificação, optou-se pela realização de termorretificações com injeção de nitrogênio gasoso no interior da carga para os tratamentos com temperaturas acima de $160{ }^{\circ} \mathrm{C}$.

\section{Avaliação da perda de massa}

Antes das termorretificações, as amostras foram secas em estufa a $100{ }^{\circ} \mathrm{C}$ até atingirem massa constante. As massas secas das amostras foram registradas antes e após as termorretificações, de modo a permitir o cálculo da perda de massa causada por efeito do tratamento térmico. Para a pesagem das amostras, aguardou-se o resfriamento das câmaras de termorretificação até $30^{\circ} \mathrm{C}$.

\section{Ensaios mecânicos}

Para avaliação comparativa do efeito da termorretificação nas propriedades mecânicas da madeira, foram realizados ensaios mecânicos de flexão estática (aplicando-se a carga sempre na face tangencial dos corpos de prova, sobre o lado côncavo dos anéis de crescimento), compressão paralela à grã e cisalhamento paralelo à grã.

Os ensaios mecânicos consideraram cinco corpos de prova representativos de cada tratamento. Os corpos de prova destinados ao ensaio de flexão estática tiveram dimensões de 2 × 2 x $30 \mathrm{~cm}$ (radial x tangencial x longitudinal), conforme proposto pela Comisión Panamericana de Normas Técnicas COPANT 555 (1973). Para os ensaios de compressão paralela e cisalhamento, foram preparados corpos de prova de $2 \times 2 \times 3 \mathrm{~cm}$ (radial x tangencial x longitudinal), de acordo com o Método Brasileiro - MB-26 da Associação Brasileira de Normas Técnicas (ABNT), (1940). Todos os corpos de prova foram previamente climatizados à temperatura de $22{ }^{\circ} \mathrm{C}$ e $65 \%$ de umidade relativa do ar até atingirem o equilíbrio higroscópico.

Em todos os ensaios mecânicos, os esforços foram registrados por meio de uma célula de carga com capacidade máxima de 10 toneladas, acoplada a uma máquina universal mecânica e um microcomputador. As deformações ocorridas durante os ensaios de flexão estática foram medidas por meio de um sensor de deslocamento linear (Linear Variable Differential Transformer - LVDT) apoiado no centro do corpo de prova. A determinação do módulo de ruptura (MOR) foi realizada por meio do software Pavitest, de acordo com a COPANT 555 (1973) (ensaios de flexão estática) e Norma Brasileira Regulamentadora - NBR 7190 da ABNT (1997) (ensaios de compressão paralela e cisalhamento). O Módulo de Elasticidade (MOE), obtido em flexão estática, foi calculado a partir das curvas esforçodeformação, conforme a COPANT 555 (1973).

\section{Análise estatística dos resultados}

A avaliação estatística dos resultados foi conduzida por meio de análises de variância (ANOVA) e comparações múltiplas entre médias (Teste de Tukey). As comparações entre médias referentes aos tipos de tratamento (i.e. atmosfera em presença ou escassez de oxigênio) foram realizadas somente para temperaturas máximas de $160{ }^{\circ} \mathrm{C}$ e $180^{\circ} \mathrm{C}$. O coeficiente de correlação (r) foi calculado para avaliar o grau de correlação entre os módulos (ruptura e elasticidade) e as temperaturas aplicadas.

\section{RESULTADOS E DISCUSSÃO}

\section{Perda de massa da madeira}

A tabela 1 contém os resultados de perda de massa para as madeiras de Eucalyptus grandis e Pinus caribaea var. hondurensis termorretificadas a três temperaturas máximas em presença ou escassez de oxigênio, apresentando comparações estatísticas múltiplas entre médias, para todos os fatores estudados.

A madeira de E. grandis apresentou perda de massa mais elevada $(4,89 \%)$ que aquela observada para $P$. caribaea var. hondurensis $(2,89 \%)$. Isso indica que o pínus foi menos susceptível do que o eucalipto à perda de massa em função do aumento de temperatura.

Conforme previsto, o aumento da temperatura máxima de tratamento acarretou maiores perdas de massa. Para a madeira Eucalyptus, no entanto, o aumento da temperatura de 180 para $200{ }^{\circ} \mathrm{C}$ não afetou significativamente a perda de massa (Tabela 1, C1). A madeira de Pinus apresentou perda de massa significativa apenas a partir de $180{ }^{\circ} \mathrm{C}$ (Tabela $1, \mathrm{C} 1$ ). À temperatura máxima de $200{ }^{\circ} \mathrm{C}$, as duas espécies 
apresentaram perdas de massa estatisticamente semelhantes (Tabela 1, C2). Portanto, a menor susceptibilidade à perda de massa na madeira de Pinus foi constatada apenas em temperaturas inferiores a $180^{\circ} \mathrm{C}$.

Tabela 1. Comparações estatísticas múltiplas de perda de massa das madeiras estudadas.

Table 1. Multiple statistical comparisons for wood mass loss.

\begin{tabular}{|c|c|c|c|c|c|c|c|}
\hline Espécie & Atmosfera & $\begin{array}{l}\text { Temperatura } \\
\text { máxima }\left({ }^{\circ} \mathrm{C}\right)\end{array}$ & $\begin{array}{c}\text { Perda de } \\
\text { massa }(\%)\end{array}$ & $\begin{array}{c}\text { Erro-padrão } \\
(\%)\end{array}$ & C1 & $\mathrm{C2}$ & C3 \\
\hline \multirow{6}{*}{ Eucalyptus grandis } & \multirow{4}{*}{$\begin{array}{l}\text { Presença de } \\
\text { oxigênio }\end{array}$} & 140 & 2,63 & 0,08 & $\underline{\mathrm{C}}$ & $\mathrm{A}$ & \\
\hline & & 160 & 4,03 & 0,15 & $\underline{\mathrm{B}}$ & A & $\mathrm{a}$ \\
\hline & & 180 & 5,40 & 0,28 & $\underline{\bar{A}}$ & $\mathrm{~A}$ & $\mathrm{~b}$ \\
\hline & & 160 & 2,24 & 0,17 & $\overline{\mathrm{B}}$ & A & $\mathrm{b}$ \\
\hline & \multirow{2}{*}{$\begin{array}{l}\text { Escassez de } \\
\text { oxigênio }\end{array}$} & 180 & 6,88 & 0,42 & $\underline{\bar{A}}$ & A & $\mathrm{a}$ \\
\hline & & 200 & 8,13 & 1,39 & $\overline{\mathrm{A}}$ & $\mathrm{A}$ & \\
\hline \multirow{6}{*}{$\begin{array}{l}\text { Pinus caribaea var. } \\
\text { hondurensis }\end{array}$} & \multirow{4}{*}{$\begin{array}{l}\text { Presença de } \\
\text { oxigênio }\end{array}$} & 140 & 0,62 & 0,09 & $\overline{\mathrm{B}}$ & $\mathrm{B}$ & \\
\hline & & 160 & 0,92 & 0,11 & $\underline{B}$ & B & $\mathrm{a}$ \\
\hline & & 180 & 3,33 & 0,24 & $\underline{\mathrm{A}}$ & B & $\mathrm{a}$ \\
\hline & & 160 & 1,04 & 0,10 & $\overline{\mathrm{C}}$ & $\mathrm{B}$ & $\mathrm{a}$ \\
\hline & \multirow{2}{*}{$\begin{array}{l}\text { Escassez de } \\
\text { oxigênio }\end{array}$} & 180 & 3,91 & 0,28 & $\underline{\bar{B}}$ & B & $\mathrm{a}$ \\
\hline & & 200 & 7,51 & 0,46 & $\underline{\mathrm{A}}$ & A & \\
\hline
\end{tabular}

C1: Comparações (teste de Tukey) entre temperaturas máximas, para uma mesma espécie e mesmo tipo de tratamento (presença ou escassez de oxigênio). C2: Comparações (teste de Tukey) entre espécies, para um mesmo tipo de tratamento (presença ou escassez de oxigênio) e mesma temperatura máxima. C3: Comparações (teste de Tukey) entre tipos de tratamento (presença ou escassez de oxigênio), para uma mesma espécie e mesma temperatura máxima. Essas comparações foram realizadas somente para temperaturas máximas de $160^{\circ} \mathrm{C}$ e $180^{\circ} \mathrm{C}$.

Com base na média geral dos tratamentos, a presença ou escassez de oxigênio não demonstrou efeito significativo na perda de massa. As amostras termorretificadas apresentaram perdas de massa de 3,42 e 3,52\%, na presença e escassez de oxigênio, respectivamente. De fato, para a madeira de Pinus, não foi constatado qualquer efeito significativo da presença de oxigênio sobre a perda de massa (Tabela 1, C3). Para Eucalyptus, a presença de oxigênio acarretou perda adicional de massa à temperatura máxima de $160^{\circ} \mathrm{C}$ e perda reduzida de massa à temperatura de $180^{\circ} \mathrm{C}$.

De maneira geral, os resultados sugerem a não existência de um efeito significativo do oxigênio sobre a perda de massa durante a termorretificação. Acredita-se que o oxigênio presente na câmara de termorretificação possa causar oxidação restrita à superfície das amostras, que tem contato direto com a atmosfera da câmara. Nesse contexto, a oxidação superficial resultaria em pouca ou nenhuma diferença significativa na perda de massa de uma amostra. Além disso, os lumes das células da madeira seca contêm oxigênio, o que contribui para a oxidação no interior da madeira, independentemente da composição da atmosfera na câmara de termorretificação.

A perda de massa é, reconhecidamente, um fator causador de perda de resistência mecânica da madeira. Alguns trabalhos demonstraram que o aumento das temperaturas da termorretificação proporciona, além de uma perda de massa, uma perda de resistência mecânica (STAMM, 1956; TSOUMIS, 1991; WINANDY; LEBOW, 2001; KAMDEM et al., 2002; BEKHTA; NIEMZ, 2003). Assim, tendo em vista que a presença de oxigênio na câmara de termorretificação não deve ter afetado a perda de massa, levanta-se a hipótese de que ela também não reduzirá significativamente a resistência mecânica das amostras termorretificadas.

Na prática, a injeção de nitrogênio na câmara de termorretificação, para expulsão do oxigênio, constitui-se em operação relativamente onerosa. No contexto dos resultados obtidos neste experimento, conclui-se que o emprego do nitrogênio somente seria justificável, em escala industrial, com o propósito de prevenção de incêndios. Nesse caso, seria recomendável a aquisição de equipamentos para produção própria (isolamento) do nitrogênio.

\section{Propriedades mecânicas das espécies estudadas}

De maneira geral, a termorretificação resultou em queda das resistências à flexão e cisalhamento. O Eucalyptus, que apresentou maior susceptibilidade à perda de massa com o aumento da temperatura, também manifestou maior propensão à perda de resistência em função do tratamento térmico. Em Pinus, 
no entanto, apenas a resistência ao cisalhamento decresceu em função do tratamento térmico, à temperatura de $200^{\circ} \mathrm{C}$.

Dentre as propriedades mecânicas ensaiadas, a resistência à compressão paralela à grã foi a menos afetada pelas temperaturas máximas de termorretificação. Para as duas espécies estudadas, a temperatura máxima aplicada $\left(200^{\circ} \mathrm{C}\right)$ não foi suficiente para provocar perda significativa da resistência à compressão paralela.

Observou-se uma tendência de incremento do módulo MOE em função do aumento da temperatura máxima da termorretificação. Isso indica que a madeira termorretificada tende a aumentar sua capacidade de se opor a deformações elásticas quando sujeita a solicitações mecânicas, diminuindo a flecha que precede a ruptura, apresentando rupturas mais frágeis (menos dúcteis) que aquelas observadas em madeiras não tratadas. Esse efeito foi constatado apenas em Eucalyptus.

Os resultados não permitem conclusões claras quanto ao efeito da presença de oxigênio durante a termorretificação sobre as propriedades mecânicas da madeira tratada. Conforme indicado em discussões anteriores, é provável que, no intervalo de temperaturas testadas, a presença do oxigênio tenha efeito apenas superficial nas pranchas tratadas termicamente.

A tabela 2 contém os resultados de MOE, obtidos em flexão estática, para Eucalyptus grandis e Pinus caribaea var. hondurensis, termorretificados a três temperaturas máximas em presença ou escassez de oxigênio, apresentando comparações estatísticas múltiplas entre médias, para todos os fatores estudados.

Tabela 2. Comparações estatísticas múltiplas do MOE, obtidos em flexão estática, apresentados em MPa. Table 2. Multiple statistical comparisons for MOE obtained in static bending, presented in MPa.

\begin{tabular}{|c|c|c|c|c|c|c|c|}
\hline Espécie & Atmosfera & $\begin{array}{l}\text { Temperatura } \\
\text { máxima }\left({ }^{\circ} \mathrm{C}\right)\end{array}$ & MOE (MPa) & Erro-padrão & C1 & $\mathrm{C} 2$ & C3 \\
\hline \multirow{8}{*}{ Eucalyptus grandis } & \multirow{4}{*}{$\begin{array}{l}\text { Presença de } \\
\text { oxigênio }\end{array}$} & Testemunha & 13.587 & 503 & $\underline{\mathrm{B}}$ & A & \\
\hline & & 140 & 15.064 & 753 & $\underline{\mathrm{AB}}$ & A & \\
\hline & & 160 & 16.483 & 645 & $\underline{\mathrm{A}}$ & A & $\mathrm{a}$ \\
\hline & & 180 & 14.597 & 541 & $\overline{\mathrm{AB}}$ & A & a \\
\hline & \multirow{4}{*}{$\begin{array}{l}\text { Escassez de } \\
\text { oxigênio }\end{array}$} & Testemunha & 13.221 & 208 & $\overline{\mathrm{A}}$ & A & \\
\hline & & 160 & 14.241 & 520 & $\underline{\mathrm{A}}$ & A & $\mathrm{b}$ \\
\hline & & 180 & 15.018 & 1.269 & $\underline{\mathrm{A}}$ & A & $\mathrm{a}$ \\
\hline & & 200 & 16.686 & 1.059 & $\underline{\mathrm{A}}$ & A & \\
\hline \multirow{8}{*}{$\begin{array}{l}\text { Pinus caribaea var. } \\
\text { hondurensis }\end{array}$} & \multirow{4}{*}{$\begin{array}{l}\text { Presença de } \\
\text { oxigênio }\end{array}$} & Testemunha & 9.623 & 2.414 & $\underline{A}$ & $\mathrm{~B}$ & \\
\hline & & 140 & 10.332 & 1.289 & $\underline{\mathrm{A}}$ & $\mathrm{B}$ & \\
\hline & & 160 & 10.337 & 1.350 & $\underline{\mathrm{A}}$ & $\mathrm{B}$ & a \\
\hline & & 180 & 9.818 & 1.508 & $\underline{\mathrm{A}}$ & $\mathrm{B}$ & $\mathrm{a}$ \\
\hline & \multirow{4}{*}{$\begin{array}{l}\text { Escassez de } \\
\text { oxigênio }\end{array}$} & Testemunha & 9.114 & 293 & $\underline{A}$ & $\mathrm{~B}$ & \\
\hline & & 160 & 10.052 & 1.849 & $\underline{A}$ & A & $\mathrm{a}$ \\
\hline & & 180 & 10.985 & 1.148 & $\underline{\mathrm{A}}$ & $\mathrm{B}$ & $\mathrm{a}$ \\
\hline & & 200 & 11.837 & 1.204 & $\underline{\mathrm{A}}$ & $\mathrm{B}$ & \\
\hline
\end{tabular}

C1: Comparações (teste de Tukey) entre temperaturas máximas, para uma mesma espécie e mesmo tipo de tratamento (presença ou escassez de oxigênio). C2: Comparações (teste de Tukey) entre espécies, para um mesmo tipo de tratamento (presença ou escassez de oxigênio) e mesma temperatura máxima. C3: Comparações (teste de Tukey) entre tipos de tratamento (presença ou escassez de oxigênio), para uma mesma espécie e mesma temperatura máxima. Essas comparações foram realizadas somente para temperaturas máximas de $160^{\circ} \mathrm{C}$ e $180^{\circ} \mathrm{C}$.

O MOE, obtido em flexão estática, foi mais elevado em Eucalyptus (14.862 MPa) do que em Pinus (10.262 MPa), o que indica um comportamento mais dúctil do pínus em comparação ao eucalipto. A diferença de MOE entre as espécies não foi estatisticamente confirmada apenas para o tratamento a $160{ }^{\circ} \mathrm{C}$ na escassez de oxigênio (Tabela 2, C2).

A madeira de pínus não manifestou alterações significativas do MOE em função das temperaturas máximas de tratamento (Tabela 2, C1), o que foi confirmado pela ausência de correlação entre tais variáveis para essa espécie.

Por outro lado, a madeira de eucalipto apresentou incremento do MOE à medida que a temperatura máxima da termorretificação aumentou (Tabela 2, C1). Para essa espécie, o MOE esteve positivamente correlacionado com a temperatura máxima do tratamento $(r=0,46)$. Isso indica que o 
eucalipto tratado termicamente tende a perder sua capacidade de se deformar elasticamente quando sujeito à flexão, manifestando rupturas mais frágeis que aquelas observadas na madeira não tratada. Esse comportamento corrobora resultados obtidos por Santos (2000).

As médias gerais de MOE foram similares entre as madeiras termorretificadas em presença (12.809 MPa) e escassez de oxigênio (12.574 MPa). No entanto, para Eucalyptus tratado à temperatura máxima de $160^{\circ} \mathrm{C}$, o MOE foi mais elevado em amostras tratadas na presença de oxigênio (Tabela 2, C3).

Os resultados de módulo de ruptura (MOR), obtidos em flexão estática, para Eucalyptus grandis e Pinus caribaea var. hondurensis, termorretificados a três temperaturas máximas em presença ou escassez de oxigênio, são apresentados na tabela 3, incluindo comparações estatísticas múltiplas entre médias, para todos os fatores estudados.

Embora a média geral de MOR (flexão estática) tenha sido superior em Eucalyptus (82 MPa) que em Pinus (58 MPa), essa diferença entre as médias esteve principalmente associada às amostras não tratadas (testemunhas, Tabela 3, C2). Com exceção das testemunhas, não foram detectadas diferenças significativas do MOR entre as duas espécies estudadas (Tabela 3, C2). A resistência à flexão estática tornou-se mais similar entre espécies à medida que as temperaturas aumentaram (Tabela 3).

Em geral, a resistência à flexão estática (MOR) foi negativamente correlacionada com a temperatura máxima de tratamento térmico $(r=-0,34)$. No entanto, essa correlação somente foi significativa em Eucalyptus $(\mathrm{r}=-0,56)$. As temperaturas máximas testadas não foram suficientes para alterar significativamente a resistência de Pinus à flexão (Tabela $3, \mathrm{C} 1$ ). Por outro lado, a resistência à flexão de Eucalyptus foi afetada pelas temperaturas máximas de tratamento, principalmente a partir de $180^{\circ} \mathrm{C}$. A sensibilidade do eucalipto ao aumento da temperatura é certamente atribuída a sua maior perda de massa durante o tratamento térmico.

Para as duas espécies estudadas, o MOR em flexão estática não apresentou alterações em função da presença (64 MPa) ou escassez de oxigênio (66 MPa) na câmara de termorretificação.

Tabela 3. Comparações estatísticas múltiplas do MOR, obtidos em flexão estática, apresentados em MPa. Table 3. Multiple statistical comparisons for MOR obtained in static bending, presented in MPa.

\begin{tabular}{|c|c|c|c|c|c|c|c|}
\hline Espécie & Atmosfera & $\begin{array}{l}\text { Temperatura } \\
\text { máxima }\left({ }^{\circ} \mathbf{C}\right)\end{array}$ & MOR (MPa) & Erro-padrão & $\mathrm{C} 1$ & $\mathrm{C} 2$ & C3 \\
\hline \multirow{8}{*}{ Eucalyptus grandis } & \multirow{4}{*}{$\begin{array}{l}\text { Presença de } \\
\text { oxigênio }\end{array}$} & Testemunha & 103 & 5 & $\underline{\mathrm{A}}$ & $\mathrm{A}$ & \\
\hline & & 140 & 81 & 18 & $\underline{\mathrm{AB}}$ & $\mathrm{A}$ & \\
\hline & & 160 & 88 & 9 & $\underline{\mathrm{AB}}$ & A & $\mathrm{a}$ \\
\hline & & 180 & 48 & 12 & $\underline{\mathrm{B}}$ & A & $\mathrm{a}$ \\
\hline & \multirow{4}{*}{$\begin{array}{l}\text { Escassez de } \\
\text { oxigênio }\end{array}$} & Testemunha & 112 & 2 & $\underline{\mathrm{A}}$ & A & \\
\hline & & 160 & 93 & 8 & $\underline{\mathrm{AB}}$ & $\mathrm{A}$ & $\mathrm{a}$ \\
\hline & & 180 & 56 & 14 & $\underline{\mathrm{C}}$ & A & $\mathrm{a}$ \\
\hline & & 200 & 75 & 7 & $\underline{\mathrm{BC}}$ & A & \\
\hline \multirow{8}{*}{$\begin{array}{l}\text { Pinus caribaea var. } \\
\text { hondurensis }\end{array}$} & \multirow{4}{*}{$\begin{array}{l}\text { Presença de } \\
\text { oxigênio }\end{array}$} & Testemunha & 64 & 2 & $\underline{\mathrm{A}}$ & $\mathrm{B}$ & \\
\hline & & 140 & 51 & 10 & $\underline{\mathrm{A}}$ & A & \\
\hline & & 160 & 65 & 8 & $\underline{\mathrm{A}}$ & A & $\mathrm{a}$ \\
\hline & & 180 & 54 & 12 & $\underline{\mathrm{A}}$ & A & $\mathrm{a}$ \\
\hline & \multirow{4}{*}{$\begin{array}{l}\text { Escassez de } \\
\text { oxigênio }\end{array}$} & Testemunha & 60 & 2 & $\underline{\mathrm{A}}$ & B & \\
\hline & & 160 & 64 & 14 & $\underline{\mathrm{A}}$ & A & $\mathrm{a}$ \\
\hline & & 180 & 52 & 9 & $\underline{\mathrm{A}}$ & A & $\mathrm{a}$ \\
\hline & & 200 & 57 & 10 & $\underline{\mathrm{A}}$ & A & \\
\hline
\end{tabular}

C1: Comparações (teste de Tukey) entre temperaturas máximas, para uma mesma espécie e mesmo tipo de tratamento (presença ou escassez de oxigênio). C2: Comparações (teste de Tukey) entre espécies, para um mesmo tipo de tratamento (presença ou escassez de oxigênio) e mesma temperatura máxima. C3: Comparações (teste de Tukey) entre tipos de tratamento (presença ou escassez de oxigênio), para uma mesma espécie e mesma temperatura máxima. Essas comparações foram realizadas somente para temperaturas máximas de $160^{\circ} \mathrm{C}$ e $180^{\circ} \mathrm{C}$.

A tabela 4 contém os resultados de MOR, obtidos em compressão paralela à grã, para Eucalyptus grandis e Pinus caribaea var. hondurensis, termorretificados a três temperaturas máximas em presença ou escassez de oxigênio, apresentando comparações estatísticas múltiplas entre médias, para todos os fatores estudados. 
O eucalipto mostrou-se mais resistente à compressão paralela $(57,5 \mathrm{MPa})$ que o pínus (39,8 MPa). Esse fato foi observado em todos os tratamentos estudados, exceto para a temperatura máxima de $200^{\circ} \mathrm{C}$, na qual a resistência à compressão foi estatisticamente similar para as duas espécies (Tabela 4, C2). A maior resistência de Eucalyptus à compressão se deve principalmente a sua maior densidade, comparada àquela de Pinus.

Dentre as propriedades mecânicas avaliadas, a resistência à compressão foi a menos afetada pelas temperaturas máximas de termorretificação. Não foi constatado qualquer efeito significativo ou correlação entre a temperatura máxima e essa propriedade mecânica, para as duas espécies estudadas (Tabela 4, C1). Logo, as temperaturas máximas aplicadas neste estudo não foram suficientes para comprometer o caráter estrutural da madeira, para a solicitação em compressão paralela à grã.

Tabela 4. Comparações estatísticas múltiplas dos MOR, obtidos em compressão paralela à grã, apresentados em MPa.

Table 4. Multiple statistical comparisons for MOR obtained in parallel compression loading, presented in MPa.

\begin{tabular}{|c|c|c|c|c|c|c|c|}
\hline Espécie & Atmosfera & $\begin{array}{l}\text { Temperatura } \\
\text { máxima }\left({ }^{\circ} \mathrm{C}\right)\end{array}$ & MOR (MPa) & Erro-padrão & C1 & $\mathrm{C} 2$ & C3 \\
\hline \multirow{8}{*}{ Eucalyptus grandis } & \multirow{4}{*}{$\begin{array}{l}\text { Presença de } \\
\text { oxigênio }\end{array}$} & Testemunha & 54,2 & 2,7 & $\underline{\mathrm{A}}$ & $\mathrm{A}$ & \\
\hline & & 140 & 56,6 & 2,5 & $\underline{\bar{A}}$ & A & \\
\hline & & 160 & 63,1 & 4,9 & $\underline{\mathrm{A}}$ & A & $\mathrm{a}$ \\
\hline & & 180 & 59,0 & 2,1 & $\underline{\bar{A}}$ & A & $\mathrm{a}$ \\
\hline & \multirow{4}{*}{$\begin{array}{l}\text { Escassez de } \\
\text { oxigênio }\end{array}$} & Testemunha & 55,5 & 2,3 & $\underline{\mathrm{A}}$ & A & \\
\hline & & 160 & 58,3 & 2,8 & $\underline{\mathrm{A}}$ & $\mathrm{A}$ & $\mathrm{a}$ \\
\hline & & 180 & 59,3 & 3,4 & $\underline{\mathrm{A}}$ & A & $\mathrm{a}$ \\
\hline & & 200 & 53,8 & 5,0 & $\overline{\mathrm{A}}$ & A & \\
\hline \multirow{8}{*}{$\begin{array}{l}\text { Pinus caribaea var. } \\
\text { hondurensis }\end{array}$} & \multirow{4}{*}{$\begin{array}{l}\text { Presença de } \\
\text { oxigênio }\end{array}$} & Testemunha & 36,3 & 2,9 & $\overline{\mathrm{A}}$ & $\mathrm{B}$ & \\
\hline & & 140 & 38,9 & 4,0 & $\underline{\mathrm{A}}$ & $\mathrm{B}$ & \\
\hline & & 160 & 42,4 & 3,2 & $\underline{\mathrm{A}}$ & $\mathrm{B}$ & $\mathrm{a}$ \\
\hline & & 180 & 40,4 & 4,0 & $\underline{\bar{A}}$ & $\mathrm{~B}$ & $\mathrm{a}$ \\
\hline & \multirow{4}{*}{$\begin{array}{c}\text { Escassez de } \\
\text { oxigênio }\end{array}$} & Testemunha & 33,6 & 0,9 & $\underline{\bar{A}}$ & $\mathrm{~B}$ & \\
\hline & & 160 & 42,5 & 4,4 & $\underline{\bar{A}}$ & $\mathrm{~B}$ & $\mathrm{a}$ \\
\hline & & 180 & 42,5 & 2,6 & $\underline{\mathrm{A}}$ & $\mathrm{B}$ & a \\
\hline & & 200 & 41,6 & 4,9 & $\underline{\overline{\mathrm{A}}}$ & $\mathrm{A}$ & \\
\hline
\end{tabular}

C1: Comparações (teste de Tukey) entre temperaturas máximas, para uma mesma espécie e mesmo tipo de tratamento (presença ou escassez de oxigênio). C2: Comparações (teste de Tukey) entre espécies, para um mesmo tipo de tratamento (presença ou escassez de oxigênio) e mesma temperatura máxima. C3: Comparações (teste de Tukey) entre tipos de tratamento (presença ou escassez de oxigênio), para uma mesma espécie e mesma temperatura máxima. Essas comparações foram realizadas somente para temperaturas máximas de $160^{\circ} \mathrm{C}$ e $180^{\circ} \mathrm{C}$.

A resistência à compressão paralela à grã também não foi alterada em função da presença (51,2 MPa) ou escassez de oxigênio $(50,7 \mathrm{MPa})$ na câmara de termorretificação. Esse fato reforça a hipótese de que, para o intervalo de temperaturas máximas testadas, a presença do oxigênio teve efeitos apenas superficiais sobre as propriedades das pranchas tratadas.

Os resultados de MOR obtidos em cisalhamento, para as madeiras de Eucalyptus grandis e Pinus caribaea var. hondurensis termorretificadas a três temperaturas máximas em presença ou escassez de oxigênio, são apresentados na tabela 5, que compila as comparações estatísticas múltiplas entre médias, para todos os fatores estudados.

As médias gerais de resistência ao cisalhamento foram similares entre o eucalipto $(10,2 \mathrm{MPa})$ e o pínus $(9,7 \mathrm{MPa})$. Para as testemunhas e amostras tratadas a $160^{\circ} \mathrm{C}$ em presença de oxigênio, no entanto, a resistência ao cisalhamento foi mais elevada em Eucalyptus que em Pinus (Tabela 5, C2). As amostras de Eucalyptus apenas foram menos resistentes que as de Pinus para o tratamento a $180{ }^{\circ} \mathrm{C}$ em presença de oxigênio.

A resistência ao cisalhamento foi a propriedade mecânica mais afetada pela temperatura de termorretificação $(r=-0,41)$. Em Pinus, esta foi a única propriedade mecânica significativamente reduzida pela ação da temperatura. Ainda assim, o pínus manifestou redução da resistência ao cisalhamento apenas para o tratamento a $200{ }^{\circ} \mathrm{C}$ (Tabela 5, C1). Além disso, para Pinus, não foi 
constatada correlação entre a temperatura máxima de tratamento e essa resistência. Por outro lado, o eucalipto sofreu queda dessa resistência a partir de 160 e $180{ }^{\circ} \mathrm{C}$, para os tratamentos em escassez e presença de oxigênio, respectivamente (Tabela 5, C1). Para Eucalyptus, a resistência ao cisalhamento foi negativamente correlacionada com a temperatura máxima da termorretificação $(r=-0,64)$.

Embora as médias gerais de resistência ao cisalhamento sejam semelhantes entre amostras tratadas em presença (10,0 MPa) e escassez (9,6 MPa) de oxigênio, os resultados obtidos em Eucalyptus não seguem um padrão definido (Tabela 5, C3). Em Pinus, a resistência ao cisalhamento não apresentou qualquer alteração em função da presença ou escassez de oxigênio na câmara de termorretificação.

Tabela 5. Comparações estatísticas múltiplas do MOR, obtidos em cisalhamento, apresentados em MPa. Table 5. Multiple statistical comparisons for MOR obtained in shear loading, presented in MPa.

\begin{tabular}{|c|c|c|c|c|c|c|c|}
\hline Espécie & Atmosfera & $\begin{array}{l}\text { Temperatura } \\
\text { máxima }\left({ }^{\circ} \mathrm{C}\right)\end{array}$ & MOR (MPa) & Erro-padrão & C1 & $\mathrm{C} 2$ & C3 \\
\hline \multirow{8}{*}{ Eucalyptus grandis } & \multirow{4}{*}{$\begin{array}{c}\text { Presença de } \\
\text { oxigênio }\end{array}$} & Testemunha & 12,7 & 0,9 & $\underline{\mathrm{A}}$ & $\mathrm{A}$ & \\
\hline & & 140 & 11,0 & 1,0 & $\underline{\bar{A}}$ & A & \\
\hline & & 160 & 12,8 & 0,6 & $\underline{\bar{A}}$ & A & $\mathrm{a}$ \\
\hline & & 180 & 6,4 & 0,5 & $\underline{B}$ & $\mathrm{~B}$ & $\mathrm{~b}$ \\
\hline & \multirow{4}{*}{$\begin{array}{c}\text { Escassez de } \\
\text { oxigênio }\end{array}$} & Testemunha & 12,7 & 0,2 & $\underline{\bar{A}}$ & A & \\
\hline & & 160 & 9,5 & 0,6 & $\underline{\mathrm{B}}$ & A & $b$ \\
\hline & & 180 & 8,6 & 0,8 & $\underline{\mathrm{B}}$ & A & $\mathrm{a}$ \\
\hline & & 200 & 8,0 & 0,5 & $\underline{\overline{\mathrm{B}}}$ & A & \\
\hline \multirow{8}{*}{$\begin{array}{l}\text { Pinus caribaea var. } \\
\text { hondurensis }\end{array}$} & \multirow{4}{*}{$\begin{array}{c}\text { Presença de } \\
\text { oxigênio }\end{array}$} & Testemunha & 10,3 & 0,4 & $\underline{\mathrm{A}}$ & $\mathrm{B}$ & \\
\hline & & 140 & 9,5 & 1,0 & $\underline{\bar{A}}$ & A & \\
\hline & & 160 & 10,1 & 0,2 & $\underline{\bar{A}}$ & $\mathrm{~B}$ & $\mathrm{a}$ \\
\hline & & 180 & 10,7 & 0,4 & $\underline{\bar{A}}$ & A & $\mathrm{a}$ \\
\hline & \multirow{4}{*}{$\begin{array}{c}\text { Escassez de } \\
\text { oxigênio }\end{array}$} & Testemunha & 9,0 & 0,5 & $\underline{\mathrm{AB}}$ & $\mathrm{B}$ & \\
\hline & & 160 & 10,1 & 0,8 & $\overline{\mathrm{AB}}$ & A & $\mathrm{a}$ \\
\hline & & 180 & 10,3 & 0,9 & $\underline{\mathrm{A}}$ & A & $\mathrm{a}$ \\
\hline & & 200 & 7,4 & 0,5 & $\underline{\mathrm{B}}$ & A & \\
\hline
\end{tabular}

C1: Comparações (teste de Tukey) entre temperaturas máximas, para uma mesma espécie e mesmo tipo de tratamento (presença ou escassez de oxigênio). C2: Comparações (teste de Tukey) entre espécies, para um mesmo tipo de tratamento (presença ou escassez de oxigênio) e mesma temperatura máxima. C3: Comparações (teste de Tukey) entre tipos de tratamento (presença ou escassez de oxigênio), para uma mesma espécie e mesma temperatura máxima. Essas comparações foram realizadas somente para temperaturas máximas de $160^{\circ} \mathrm{C}$ e $180^{\circ} \mathrm{C}$.

\section{CONCLUSÕES}

- A termorretificação acarretou perdas de massa e de resistência mecânica nas madeiras tratadas. Os efeitos do aumento da temperatura foram mais pronunciados na madeira de Eucalyptus do que em Pinus. De fato, a menor perda de massa na madeira de pínus também se refletiu em menor perda de resistência mecânica frente ao tratamento térmico.

- Na faixa de temperaturas testada (até $200{ }^{\circ} \mathrm{C}$ ) não houve comprometimento da resistência à compressão nas espécies estudadas. Portanto, a madeira termorretificada apresenta potencial para usos estruturais em que seja solicitada em compressão paralela, sem necessidades de maiores adaptações dimensionais da estrutura.

- Não é recomendável o uso das madeiras tratadas termicamente em situações nas quais a resistência ao cisalhamento seja um fator importante, a menos que sejam realizadas adaptações que confiram maior segurança à estrutura. A perda de resistência ao cisalhamento frente ao tratamento térmico foi mais pronunciada em Eucalyptus que em Pinus, o que inspira cuidados maiores no uso do eucalipto termicamente tratado.

- Para conferir maior segurança às estruturas compostas por madeiras termorretificadas, recomenda-se que a secção transversal dos componentes termorretificados sujeitos a solicitações de flexão sejam dimensionados em função das perdas de resistência mecânica. Portanto, são necessários cuidados adicionais no dimensionamento de componentes estruturais termorretificados acima de $180{ }^{\circ} \mathrm{C}$ que sejam solicitados em flexão ou cisalhamento. 


\section{AGRADECIMENTOS}

Este trabalho contou com o apoio técnico de A W Faber Castell S.A. e do Serviço Nacional de Aprendizagem Industrial "Mário Henrique Simonsen" (SENAI), além das contribuições de Adriana Maria Nolasco, Alex Canale e Luís Eduardo Facco. O trabalho foi realizado com fundos da Fundação de Amparo à Pesquisa do Estado de São Paulo (FAPESP).

\section{REFERÊNCIAS}

ASSOCIAÇÃO BRASILEIRA DE NORMAS TÉCNICAS (ABNT). MB-26. Ensaios físicos e mecânicos de madeiras. Rio de Janeiro: ABNT, 1940. 8 p.

NBR 7190. Projeto de estruturas de madeira. Anexo B - Determinação das propriedades das madeiras para projetos de estruturas. Rio de Janeiro: ABNT, 1997. 107 p.

AWOYEMI, L.; WESTERMARK, U. Effects of borate impregnation on the response of wood strength to heat treatment. Wood Science and Technology, v. 39, p. 484 - 491, 2005.

BEKHTA, P.; NIEMZ, P. Effect of high temperature on the change in color, dimensional stability and mechanical properties of spruce wood. Holzforschung, v. 57, n. 5, p. 539 - 546, 2003.

COPANT - Comisión Panamericana de Normas Técnicas. Maderas: Método de ensayo de flexión estática. COPANT 555, 1973. $11 \mathrm{p}$.

DUCHEZ, L.; GUYONNET, R. Principles and applications of wood rectification. In: WORLD CONFERENCE ON TIMBER ENGINEERING, 5, Lausanne, 1998. Proceedings... Lausanne, 1998.

KAMDEM, D. P.; PIZZI, A.; JERMANNAUD, A. Durability of heat-treated wood. Holz als Roh- und Werkstoff, v. 60, n. 1, p. 1 - 6, 2002.

KUBOJIMA, Y.; OKANO, T; OHTA, M. Bending strength and toughness of heat-treated wood. Journal of Wood Science, v. 46, n. 1, p. 8 - 15, 2000.

MITCHELL, P. H. Irreversible property changes of small loblolly pine specimens heated in air, nitrogen, or oxygen. Wood and Fiber Science, v. 20, n. 3, p. 320 - 335, 1988.

PINCELLI, A. L. P. S. M.; BRITO, J. O.; CORRENTE, J. E. Avaliação da termorretificação sobre a colagem na madeira de Eucalyptus saligna e Pinus caribaea var. hondurensis. Scientia Forestalis, n. 61, p. $122-132,2002$.

SANTOS, J. A. Mechanical behaviour of Eucalyptus wood modified by heat. Wood Science and Technology, v. 34, p. 39 - 43, 2000.

STAMM, A. J. Thermal degradation of wood and cellulose. Industrial \& Engineering Chemistry, v. 48, n. 3, p. 413 - 417, 1956.

TSOUMIS, G. Science and technology of wood: structure, properties, utilization. New York, Chapman \& Hall, 1991. 494 p.

WINANDY, J. E.; LEBOW, P. K. Modeling strength loss in wood by chemical composition. Part I. An individual component model for southern pine. Wood and Fiber Science, v. 33, n. 2, p. 239 - 254, 2001. 